\title{
Tracking corotating interaction regions from the Sun through to the orbit of Mars using ACE, MEX, VEX, and STEREO
}

\begin{abstract}
A. O. Williams, ${ }^{1}$ N. J. T. Edberg, ${ }^{1,2}$ S. E. Milan, ${ }^{1}$ M. Lester, ${ }^{1}$ M. Fränz, ${ }^{3}$ and J. A. Davies ${ }^{4}$
Received 26 May 2010; revised 16 May 2011; accepted 26 May 2011; published 23 August 2011.

[1] We study the propagation of corotating interaction regions (CIRs) through the inner heliosphere during the solar minimum of 2007-2008 using the Advanced Composition Explorer (ACE), Mars Express (MEX), Venus Express (VEX), and the Solar Terrestrial Relations Observatory (STEREO). We present observations of 24 CIRs observed during the period from 1 July 2007 to 31 August 2008. Using data from ACE, we demonstrate a method for predicting the arrival of CIRs at other locations within the heliosphere. The efficacy of the technique is assessed using observations at Mars and Venus, from MEX and VEX, respectively, and at the STEREO A and STEREO B spacecraft. We also use observations from the STEREO Heliospheric Imagers (HI) to produce another set of CIR arrival time estimates. We show that the estimated arrival times from ACE agree well with the arrival times at other spacecraft, whereas the estimates from STEREO/HI tend to agree less well. This latter difference can be explained through uncertainties in estimates due to difficulties in the unambiguous identification of the corresponding events in the STEREO/HI data.
\end{abstract}

Citation: Williams, A. O., N. J. T. Edberg, S. E. Milan, M. Lester, M. Fränz, and J. A. Davies (2011), Tracking corotating interaction regions from the Sun through to the orbit of Mars using ACE, MEX, VEX, and STEREO, J. Geophys. Res., 116, A08103, doi:10.1029/2010JA015719.

\section{Introduction}

[2] Solar wind transients, enhancements in solar wind velocity and/or density, often have effects on planetary magnetospheres that are of great interest to observers. These events are sporadic in nature and difficult to predict. However it is hoped that once they are first observed close to the Sun they can then be tracked through the solar system and their arrival at the planets predicted. These transients fall into two main categories: Coronal Mass Ejections (CMEs) and their interplanetary counterparts (ICMEs) [e.g., Richardson and Cane, 1995; Wang et al., 2005] and Corotating Interaction Regions (CIRs) [e.g., Gosling and Pizzo, 1999; Lee, 2000; Cranmer, 2002]. During the recent solar minimum of 2007/8, observations of a number of CIRs have been reported [e.g., Mason et al., 2009] and these form the focus of this study. CIRs are regions of enhanced density formed at the interface between a fast solar wind stream catching up with a preceding slower solar wind stream. As this interface rotates with the Sun the high speed stream

\footnotetext{
${ }^{1}$ Department of Physics and Astronomy, University of Leicester, Leicester, UK.

${ }^{2}$ Now at Swedish Institute of Space Physics, Uppsala, Sweden.

${ }^{3}$ Max Planck Institute for Solar System Research, Katlenburg-Linden, Germany.

${ }^{4}$ STFC Rutherford Appleton Laboratory, Didcot, UK.

Copyright 2011 by the American Geophysical Union. 0148-0227/11/2010JA015719
}

sweeps out a large swath in heliospheric longitude and the CIR assumes the shape of an Archimedean spiral, similar to the Parker spiral of the interplanetary magnetic field [Gosling and Pizzo, 1999]. CIRs are characterised in in situ measurements by a peak in plasma density followed by an increase in solar wind speed and plasma temperature. One of the objectives of the Solar Terrestrial Relations Observatory (STEREO), and more specifically the onboard Heliospheric Imagers (HI), is to trace the visible evolution of CMEs through the inner heliosphere. Due to the solar minimum conditions in which it has been operating it has also been possible to study the evolution of CIRs through the inner heliosphere using STEREO/HI [Rouillard et al., 2008, 2009a; Sheeley et al., 2008a, 2008b; Tappin and Howard, 2009; Wood et al., 2010].

[3] As previously mentioned the impact of transient solar wind events is often of great interest to observers of planetary magnetospheres. For example, solar wind transients are responsible for producing geomagnetic storms at Earth [Cliver, 2006; Milan et al., 2009] and have a demonstrable effect on other solar system bodies including Mars. At Earth the direction of the interplanetary magnetic field (IMF) as well as the dynamic pressure has an effect on the magnetosphere, whereas at Mars, pressure pulses in the solar wind have been observed to increase the outflow of atmospheric heavy ions by a factor of 2.5 [Edberg et al., 2010], and at Venus, ICMEs have also been observed to increase atmospheric ion loss [Luhmann et al., 2008a]. The role of the 


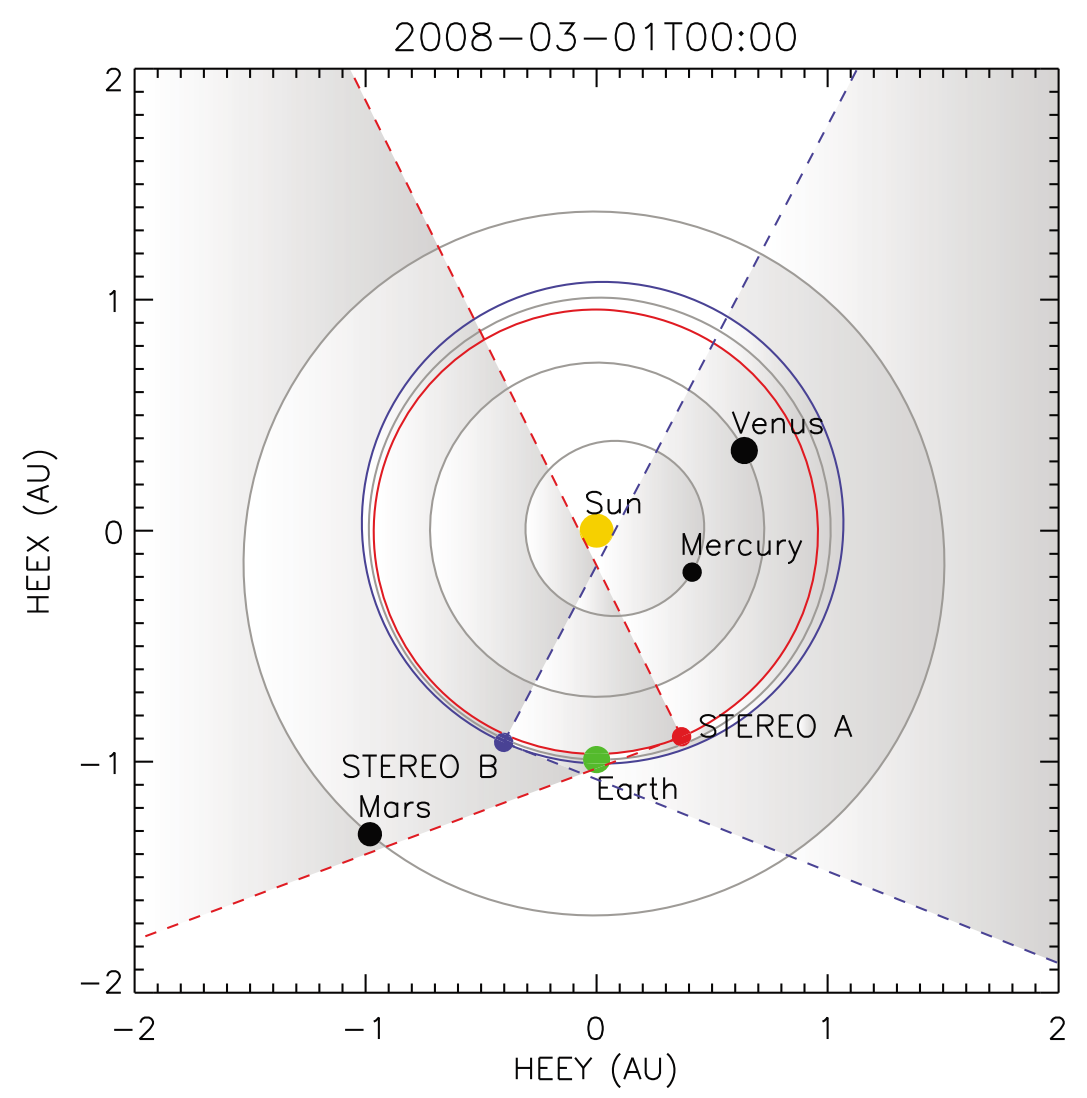

Figure 1. Location of STEREO Ahead (A: red) and Behind (B: blue) in the ecliptic plane on 1 March 2008, illustrating the extent of the HI fields of view. Also shown are the positions of the planets Mercury, Venus, Earth, and Mars and their respective orbits.

IMF in determining disturbances at Mars and Venus is less clear as these planets do not possess intrinsic magnetospheres. Prediction of the arrival times of solar wind transients at points of interest would be of great use to observers for estimating solar wind conditions if in situ measurements are unavailable. To this end, in this study we track CIRs through the inner heliosphere based on in situ and imaging observations from spacecraft situated throughout the inner solar system and then predict their arrival at different planetary bodies and spacecraft based on this information.

\section{Instrumentation}

[4] The two STEREO spacecraft were launched into heliocentric orbits on 26 October 2006. Each spacecraft was launched into an orbit such that the spacecraft would separate from Earth at a rate of approximately $22^{\circ}$ per year. The STEREO Ahead (STEREO A) spacecraft leads the Earth in its orbit, while STEREO Behind (STEREO B) lags the Earth in its orbit. Each spacecraft carries a variety of in situ instruments to monitor the solar wind and the Sun Earth Connection Coronal and Heliospheric Investigation (SECCHI) [Howard et al., 2008] suite of imagers, designed to study the Sun and the inner heliosphere. This study uses the SECCHI Heliospheric Imagers (HI) [Eyles et al., 2009]. Each spacecraft houses two of these visible-light cameras, HI- 1 and HI-2. The HI- 1 camera has a $20^{\circ}$ by $20^{\circ}$ field of view centered at $14^{\circ}$ elongation (angular distance from the
Sun) in the ecliptic plane; the HI- 2 camera has a $70^{\circ}$ by $70^{\circ}$ field of view centered at $53.7^{\circ}$. Figure 1 shows the location of the two STEREO spacecraft, and the extent of combined fields of view of the HI cameras in the ecliptic plane for each spacecraft on 1 March 2008. HI images comprise the line-of-sight integrated Thomson scattered sunlight from electron density features in the inner heliosphere, as well as the F-corona, background stars and other planets in the solar system. The HI-1 camera produces an image every 40 minutes while $\mathrm{HI}-2$ produces an image every 120 minutes.

[5] In addition to the STEREO/HI instruments, we also present in situ measurements from the STEREO Plasma and Suprathermal Ion Composition (PLASTIC) [Galvin et al., 2008] and In situ Measurements of Particles and CME Transients (IMPACT) packages [Luhmann et al., 2008b], along with observations from the Solar Wind Electron Proton and Alpha Monitor (SWEPAM) [McComas et al., 1998] and magnetic field (MAG) [Smith et al., 1998] instruments aboard the Advanced Composition Explorer (ACE) spacecraft. ACE is in a Lissajous orbit around the L1 Lagrange point, approximately 1.5 million $\mathrm{km}$ upstream of the Earth in the solar wind. This enables it to take continuous measurements of the upstream solar wind. We also present observations from the Electron Sensor (ELS) and Ion Mass Analyzer (IMA) components of the Analyzer of Space Plasmas and Energetic Atoms 3 and 4 (ASPERA-3 and ASPERA-4) instruments on board Mars Express (MEX) 
[Barabash et al., 2006] and Venus Express (VEX) [Barabash et al., 2007], respectively. ELS within ASPERA3 (ASPERA-4) measures electrons in the energy range $1 \mathrm{eV}$ to $20 \mathrm{keV}(1 \mathrm{eV}$ to $15 \mathrm{keV})$ with a field of view of $4^{\circ}$ by $360^{\circ}\left(10^{\circ}\right.$ by $\left.360^{\circ}\right)$ and a time resolution of $4 \mathrm{~s}(32 \mathrm{~s})$. IMA within ASPERA-3 and ASPERA-4 measures ions in 32 mass rings and 16 radial sectors over energies from $30 \mathrm{keV}$ to $10 \mathrm{eV}$ in 96 logarithmically equidistant steps. It then sweeps over polar angles from $-45^{\circ}$ to $+45^{\circ}$ in 16 steps, with the whole process taking $192 \mathrm{~s}$. VEX uses a similar design and instrument payload to MEX. The orbit of VEX is elliptical in such a way that it only spends a limited time of each orbit within the exosphere of Venus and for the majority of its orbit, it is in the solar wind. ASPERA-4 mainly makes observations near perigee when VEX is within the exosphere; this contributes to the apparent gaps in the ELS data we present here.

\section{Method}

[6] The plasma and magnetic field data from ACE for the period 1 July 2007 to 31 August 2008 were searched for signatures of CIRs. The signatures we used were regions of high solar wind density, greater than 15 protons $\mathrm{cm}^{-3}$, immediately followed by regions of increased solar wind speed, increasing by a factor of 1.3 or more over a 24 hour period, and increased magnetic field strength, here increasing to over $8 \mathrm{nT}$. These signatures were then confirmed by visual inspection to ensure that they were clearly CIRs and other transient structures such as CMEs were removed. A time of arrival was chosen that corresponded to the time of the first peak in density in the compressed region checked against the list presented by Mason et al. [2009].

[7] Upon finding a signature which could clearly be identified as a CIR, we found the arithmetic mean velocity from the maximum of the high speed stream following the CIR and the minimum of the low speed stream preceding it and took this as being representative of the radial velocity of the CIR, $V_{r}$. We assumed that the adjacent sources of fast and slow solar wind would continue to emit coronal plasma at these same velocities. Using this assumption, we can use the following equation to calculate the expected travel time, $\Delta t$, from the observation of the CIR at the ACE spacecraft to its impact at a planetary body or another spacecraft at a different location based on the method used by Vennerstrom et al. [2003]:

$$
\Delta t=\frac{\delta r}{V_{r}}+\frac{\delta \beta}{\omega_{\text {sun }}}
$$

where $\delta r$ is the radial distance between the two bodies of interest, which is assumed constant for the duration taken for the CIR to propagate between the two points, and $\delta \beta$ is the angle between the two bodies in terms of solar longitude, again assumed to be constant for the duration of the event. Here $\omega_{\text {sun }}$ is the equatorial rotation rate of the Sun, approximately $14.4^{\circ}$ day $^{-1}$, equivalent to a complete solar rotation every 25 days. Using equation (1) and knowledge of the time of observation of the CIR at ACE we are able to calculate an approximate arrival time of the CIR at Venus, Mars, STEREO A and STEREO B. Clearly this will not be the same plasma as observed at ACE, unless there is radial alignment, but plasma that is part of the same overall CIR front. We used these estimated arrival times to look for signs of CIR impact at Venus and Mars. Here we use 2 signatures which could indicate the arrival of a CIR, first an increased differential energy fluxes in the ELS instruments on MEX and VEX. This was previously attributed to increased electron fluxes observed in the magnetosheath to the arrival of a solar wind pressure pulse at Mars by Edberg et al. [2010]. Secondly, we can monitor the solar wind speed more directly by examining the energy of the ion beam summed over all anodes of the IMA instrument on VEX and MEX, for this we use the IMA extra data as it is the best quality available. When an increase in the beam energy is seen outside of the planetary exosphere we interpret this as an increase in the solar wind plasma speed, indicative of the arrival of a CIR. The former method is primarily used for observations by MEX and is supported by the latter method, while observations by VEX are much clearer with the IMA instrument so the latter method is preferred.

[8] We also compared in situ observations from STEREO $A$ and STEREO B with CIR observations from ACE using equation (1) to obtain an expected time of CIR impact at each spacecraft from ACE, as was done with MEX and VEX. The observations from STEREO A and STEREO B are more directly comparable with those from ACE. To obtain the observed times of arrival at STEREO A and STEREO B we compared the solar wind velocity profiles from each spacecraft using the computed cross-correlation function to obtain a time lag which best aligned the profiles from ACE with STEREO A or STEREO B.

[9] In addition, we used observations from the HI cameras aboard STEREO to aid our study of the propagation of CIRs from the Sun through to the orbit of Mars, Venus, STEREO $A$ and STEREO B. To do this we have used the technique of creating time-elongation (J-)maps, first used by Sheeley et al. [1999] with the LASCO instrument onboard SOHO and subsequently applied to the STEREO/HI observations by authors such as Rouillard et al. [2008], Davies et al. [2009], and Savani et al. [2009]. This method plots the observed intensity along a fixed solar radial, in this case the ecliptic, as a function of elongation (y axis) and time ( $\mathrm{x}$ axis). A typical example of an ecliptic J-map created using observations from the HI cameras aboard STEREO A is shown in Figure 2, for September 2007. As is evident in Figure 2 there are many tracks, with a positive gradient, indicating the path of transients traveling away from the Sun. Differenced observations are used to highlight faint propagating electron density features, so tracks are seen with a white leading edge and a black trailing edge corresponding to an increase and subsequent decrease in density. Other features visible in Figure 2 include the passage of Mercury through the field of view, starting at approximately $10^{\circ}$ elongation at the start of September and progressing through to approximately $25^{\circ}$ elongation at the end of September. Also visible are the tracks of background stars not fully removed in image processing and differencing which have a negative gradient and appear due to the motion of the spacecraft in its orbit. Finally there are some data gaps which are visible as vertical gray areas, most visible on 25 September. Figure 2 shows two clear converging families of tracks, the first entering the HI field of view between 8 and 12 September and the second entering the HI field of 


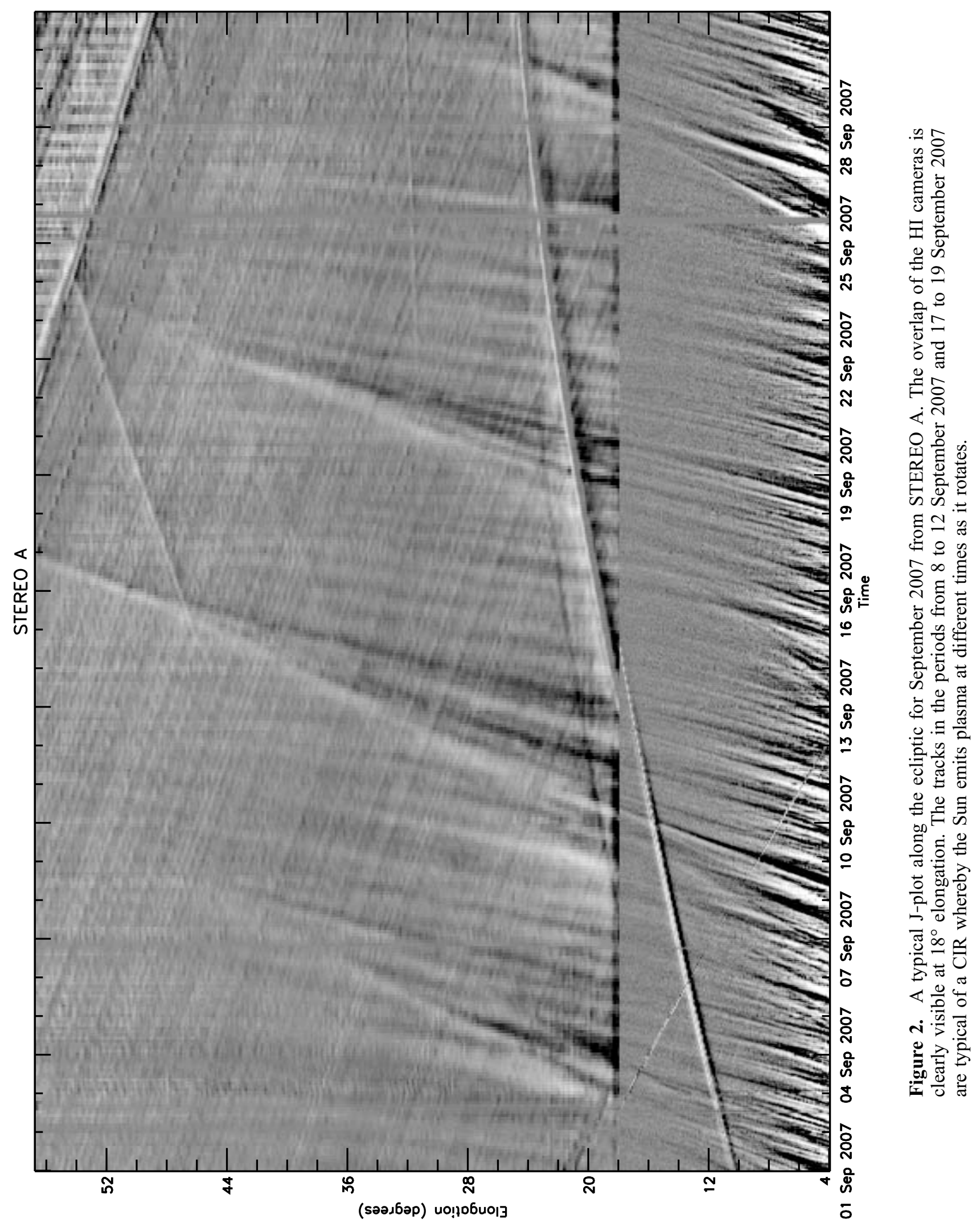


view between 17 and 19 September. Such converging families of tracks are characteristic signatures of CIRs in HI observations, and correspond to plasma elements that have been emitted by the same (rotating) source region at different times and have been entrained at the stream interface [Rouillard et al., 2009a]. Note these tracks only converge in J-maps from STEREO A; in STEREO B the family of tracks associated with a CIR diverge [Rouillard et al., 2008]. From the track of a given solar transient we can deduce its speed and direction of propagation using a technique introduced by Sheeley et al. [1999] and further developed by Rouillard et al. [2008] and Sheeley et al. [2008a, 2008b]. Its elongation-time profile, $(\alpha, \mathrm{t})$, taken from the $\mathrm{J}$-map, is used to calculate a radial propagation velocity, $V_{r}$, and angle of propagation relative to the Sun-spacecraft line, $\beta$, by fitting to

$$
\alpha(t)=\arctan \left[\frac{V_{r} t \sin \beta}{r_{A}-V_{r} t \cos \beta}\right],
$$

where $r_{A}$ is the radial distance of the observer from the Sun. The assumption that $r_{A}$ is constant for the duration of observation of the transient is used. We also assume that in the field of view of the HI instruments $V_{r}$ and $\beta$ remain constant.

[10] By fitting the elongation profile for each track with equation (2) we have built up a database of transient events each with a velocity, $V_{r}$, and direction of propagation, $\beta$. If we assume that each of these tracks is associated with a plasma parcel which forms part of a CIR, then we are then able to calculate arrival times at Venus, Earth and Mars using equation (1), substituting the appropriate values of velocity and propagation angle. For each of the CIRs identified in the ACE in situ observations we selected the most identifiable tracks and fitted them using equation (2). Of these tracks we are most interested in those which are observed to propagate to elongations greater than $30^{\circ}$. Williams et al. [2009] showed that these tracks produce estimates with smaller errors on velocity and direction of propagation.

\section{Observations}

[11] Using the methods of calculating arrival times outlined above we have examined the period from 1 July 2007 to 31 August 2008 and determined the velocities of 24 CIRs as observed by ACE. These events were selected to match times when we have good coverage of MEX data and only where the signature was clearly of a CIR. Events which were not clearly CIRs were not selected. For each of these events we looked for corresponding signatures in the data from VEX and MEX, and for observations of the groups of tracks in STEREO/HI, at the times expected based on the CIR times from ACE, using equation (1). As an example we present here a detailed study of one of these CIRs observed by ACE on the 5 January 2008 at approximately 00:30 UT. Figure 3 shows plasma data from instruments on MEX (Figures $3 \mathrm{f}-3 \mathrm{~h}$ ), VEX (Figures $3 \mathrm{a}$ and $3 \mathrm{~b}$ ), ACE (Figure 3d), STEREO A (Figure 3e) and STEREO B (Figure 3c). We present the plasma density (green line) and speed (black line) and magnetic field (red line) from the SWEPAM and MAG instruments aboard ACE at L1 (Figure 3d). For Venus and
Mars we present energy spectrograms from the ELS and IMA instruments on VEX and MEX spacecraft. At STEREO A and STEREO B we present plasma density (green line) and bulk speed (black line) observations from the PLASTIC instrument and magnetic field magnitudes (red line) from the magnetometer instrument in the IMPACT package. Using the mean of the minimum and maximum speed of the CIR observed by ACE gives $V_{r}$ of $389 \mathrm{~km} \mathrm{~s}^{-1}$. Using this value, from equation (1) we obtained estimated times of arrival of 9 January 2008 at 09:35 UT at Venus and 7 January 2008 at 03:26 UT at Mars. Similarly, expected arrival times of 6 January 2008 at 13:58 UT at STEREO A and 3 January 2008 at 07:12 UT at STEREO B were derived. Overlaid on Figure 3 are red lines indicating the observed arrival time of the CIR at each spacecraft, and black lines indicating the expected arrival times at the four other spacecraft derived from the CIR arrival time at ACE.

[12] We used the speed in a simple model of the Parker Spiral to confirm arrival times at each planet as shown in Figure 4. Figure 4 shows 5 CIR fronts produced by the model, each spiral shows the expected location of the plasma density enhancement for the indicated time in days, where $\mathrm{t}=0$ is the arrival time at ACE. Assuming a constant $V_{r}$ of $389 \mathrm{~km} \mathrm{~s}^{-1}$, the expected location of the CIR front at STEREO A $(\mathrm{t}=1.542$ days $)$, STEREO B $(\mathrm{t}=-1.292)$, Mars $(t=1.943)$ and Venus $(t=5.029)$ is shown.

[13] As the observations from VEX/ASPERA-4 and MEX/ASPERA-3 are not directly comparable with the ACE observations, for identifying CIR impact at these spacecraft we use the two methods previously explained to determine the time of arrival at the spacecraft. For VEX/ASPERA-4 this is approximately 10 January 2008 at 02:00 UT, and for MEX/ASPERA-3 this is approximately 6 January 2008 at 23:00 UT (red lines). These differ from the expected arrival times (black lines) derived from the CIR arrival time at ACE by 16 hours and 4 hours for VEX and MEX, respectively. For clarity Figure 5 shows the period from 9 to 11 January 2008 for the VEX/ELS and IMA observations. Here we can more clearly see the increase in speed of the solar wind through the increased energy of the ion beam. This shows an enhancement similar to that seen in the MEX/ELS but much shorter. Reasons for this are discussed in section 5. These enhancements in MEX/ELS have been shown to correspond to periods of increased ion outflow from the atmosphere of Mars [Edberg et al., 2010] compared to periods when no CIR is present.

[14] As mentioned in section 3 we used a cross-correlation of the velocity profiles to find the actual CIR arrival times at STEREO A and STEREO B. For the example shown in Figure 3 the arrival times from this method are 6 January 2008 at 13:30 UT for STEREO A and 3 January 2008 at 17:30 UT for STEREO B with cross-correlation values of 0.87 and 0.64 , respectively. These differ from the expected arrival times by approximately $30 \mathrm{mins}$ and 10 hours for STEREO A and STEREO B, respectively. Although in the case of STEREO B, visual inspection of the observations does indicate a density enhancement and velocity increase beginning at approximately the expected arrival time. The lower value of the cross-correlation function indicates a larger error on the timing is to be expected.

[15] As previously mentioned we have applied this technique to 24 CIRs and found in the majority of cases good 


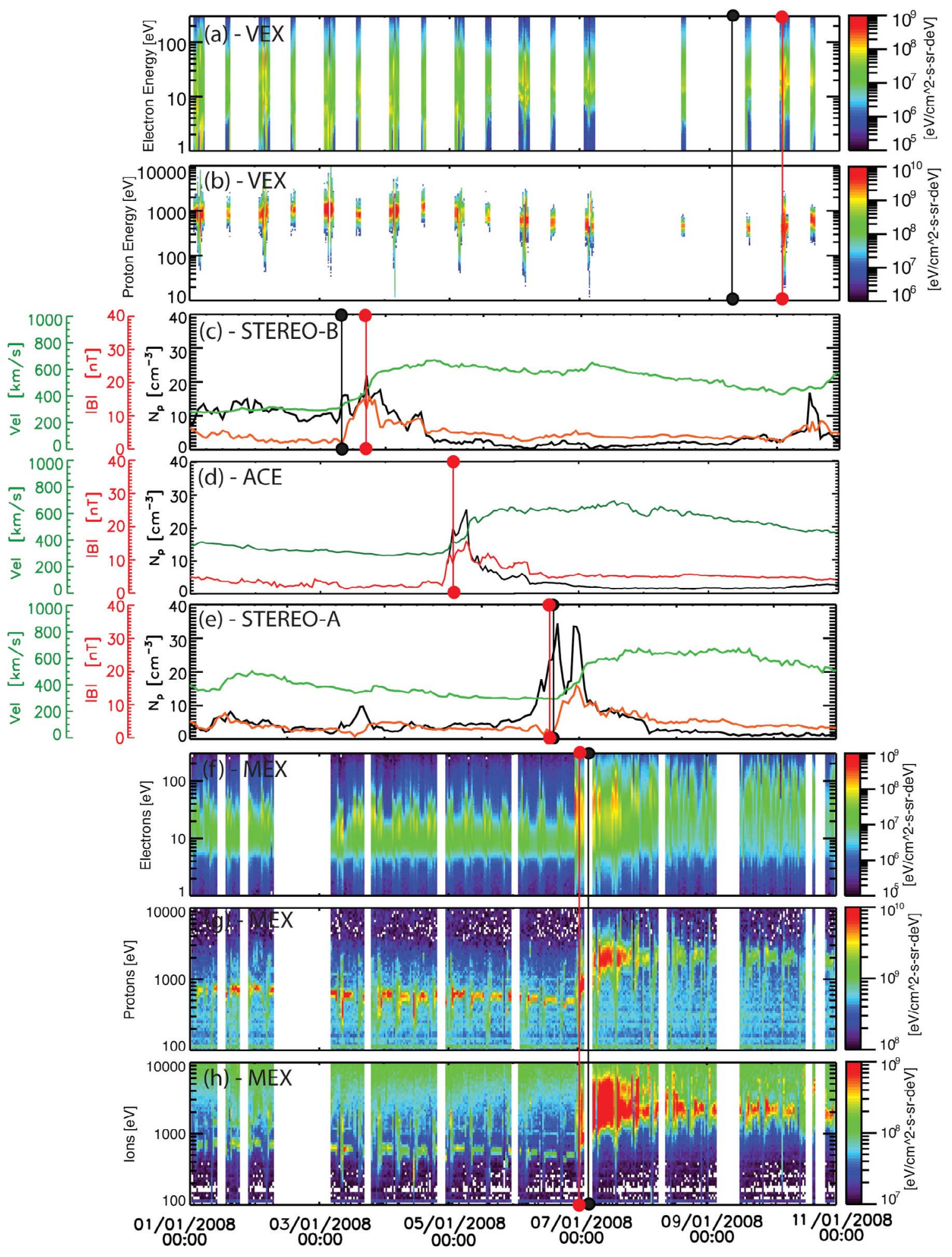

Figure 3. Time series plots of ( $a$ and b) Venus Express ELS and IMA data, (c) STEREO-B plasma and magnetometer observations, (d) ACE plasma and magnetometer observations, (e) STEREO A plasma and magnetometer observations, and $(\mathrm{f}-\mathrm{h})$ Mars Express ELS and IMA data (Figure $3 \mathrm{~h}$ is IMA "extra" data) for the period 1-11 January 2008. The observed arrival time is marked as a solid red vertical line, while the expected arrival time from the observation at ACE is marked as a solid black vertical line. 


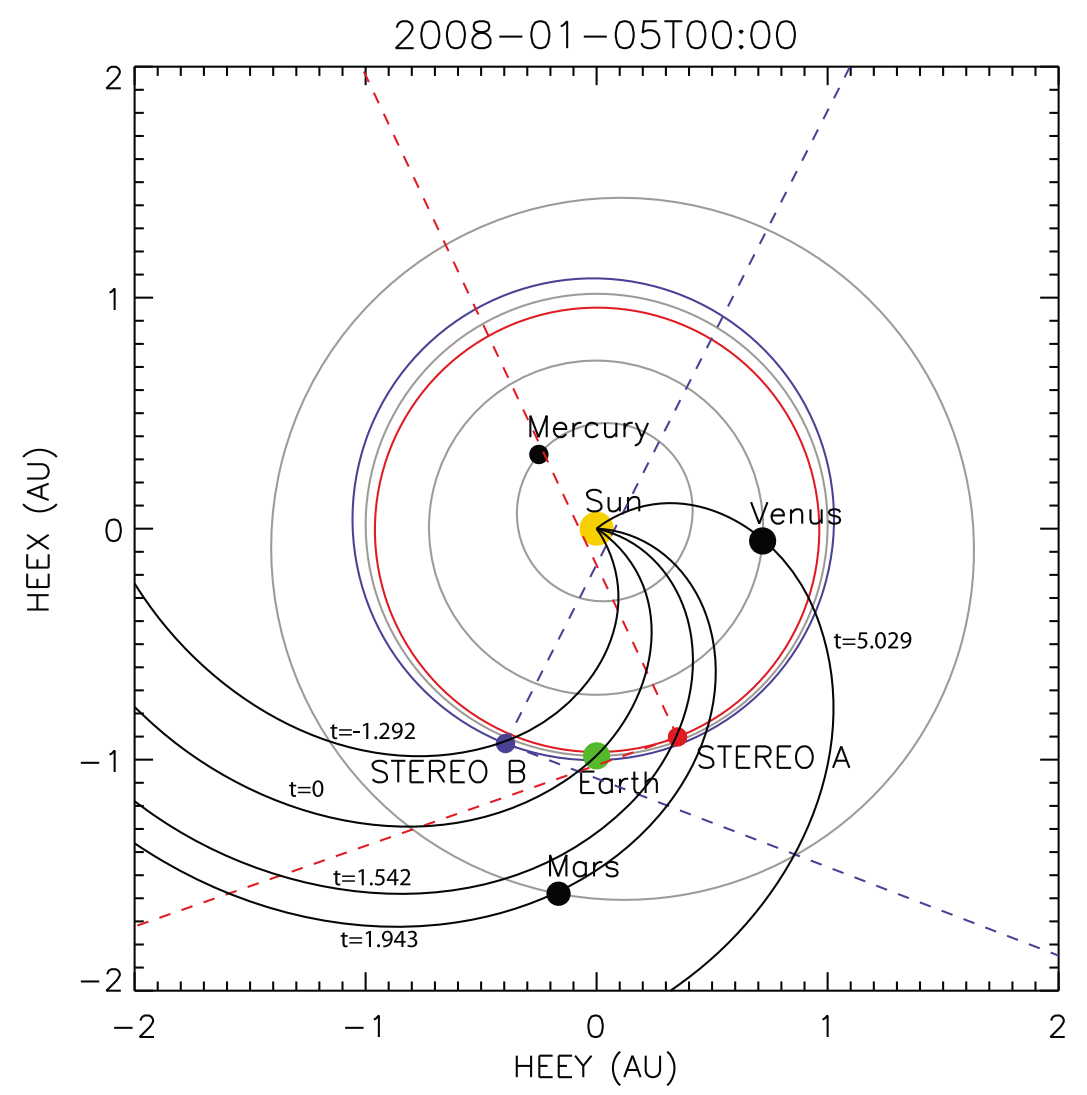

Figure 4. The location of the inner planets and STEREO A (red) and B (blue) on 5 January 2008, 00:00 UT, with the fields of view of the HI instruments marked as dashed lines. There are 5 spirals marked. The first at $t=0$ days represents the approximate Parker spiral associated with a transient which passes over Earth. All other spirals are the location of the CIR front as it passes over the other bodies, with values of $\mathrm{t}$ given in days with respect to the time from when the spiral is at Earth. Each spiral uses a velocity of $389 \mathrm{~km} \mathrm{~s}^{-1}$ to determine the shape.

correlation between estimated arrival times and observed arrival times. Figure 6 shows a sample interval (5 July 2007 to 5 August 2007) with several CIRs. Overlaid are boxes marking the observed event (blue) in VEX, ACE and MEX data with each event numbered. In some cases it should be noted that the time of arrival at VEX is between direct observations, although the arrival is clear from the difference in the energy of the solar wind ion beam between successive observations, this does lead to an error of \pm 11 hours. Here the time of arrival of events in the MEX observations are the same as reported by Edberg et al. [2010]. In addition the estimated arrival times as calculated from ACE and STEREO $\mathrm{A} / \mathrm{HI}$ are plotted, with an approximate width for error and to account for the size of the events, in green and red, respectively. Edberg et al. [2010] showed that CIRs last for approximately 36 hours for this period which enables us to add an approximate width. This is combined with the error on velocity observed by STEREO/HI as defined by Rouillard et al. [2010].

[16] In general using the ACE data gives a very good estimate for the time of arrival at other planetary bodies; this is best shown by presenting the difference between arrival times expected from $\mathrm{ACE}$ and the observed arrival time from MEX, STEREO A and STEREO B for all 24 events.

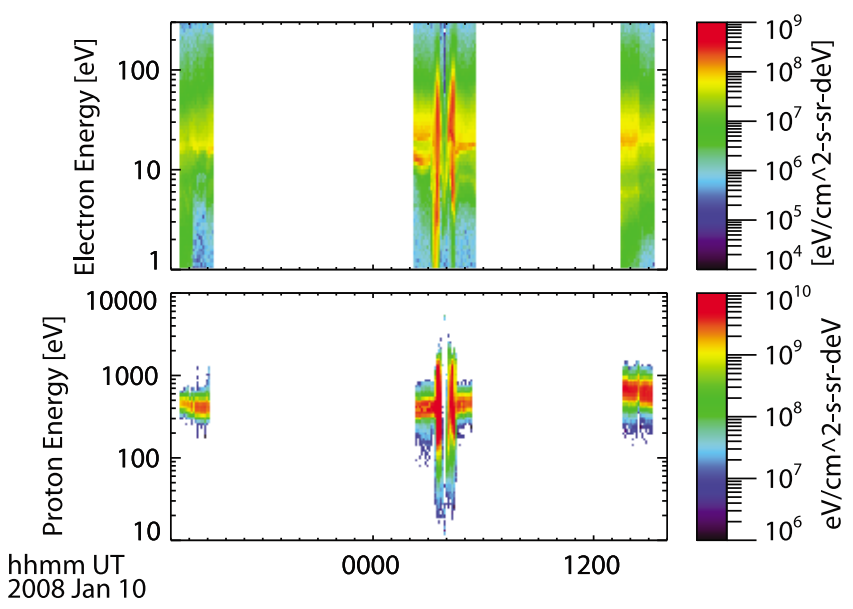

Figure 5. Exploded section of Venus Express ELS and IMA data from Figure 3 showing the enhanced electron energies from 9 to 11 January 2008. (top) The increase in the energy of the main electron beam. (bottom) A slight increase is shown in the ion beam energy, indicating an increase in the solar wind speed. 


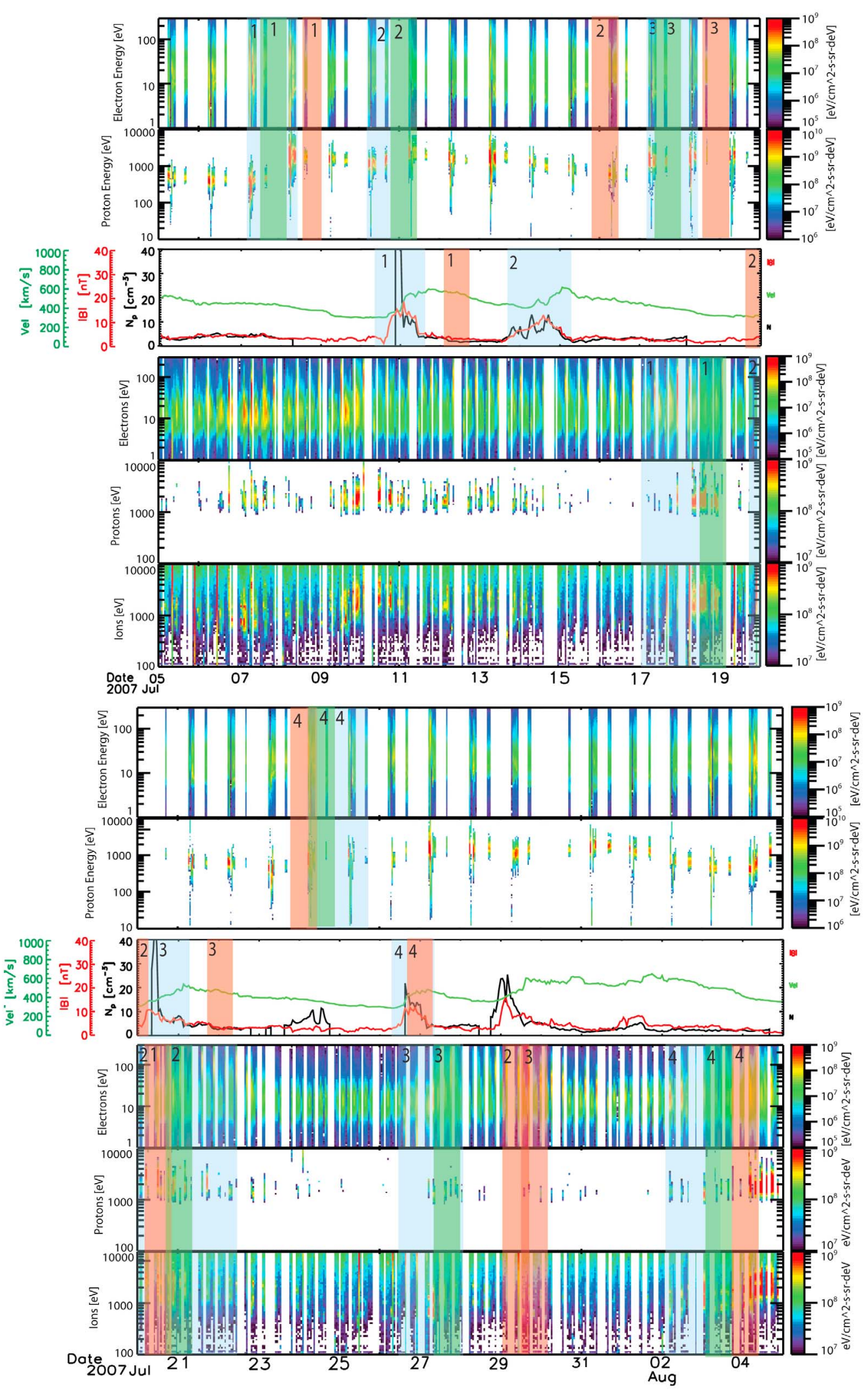

Figure 6. Time series plots as in Figure 3 for the period from 5 July 2007 to 5 August 2007. During this period, 4 CIRs are visible in VEX, ACE, and MEX. The blue boxes indicate the observations of the numbered CIR. Green boxes indicate predictions using ACE, while red boxes indicate predictions from STEREO/HI observations. 

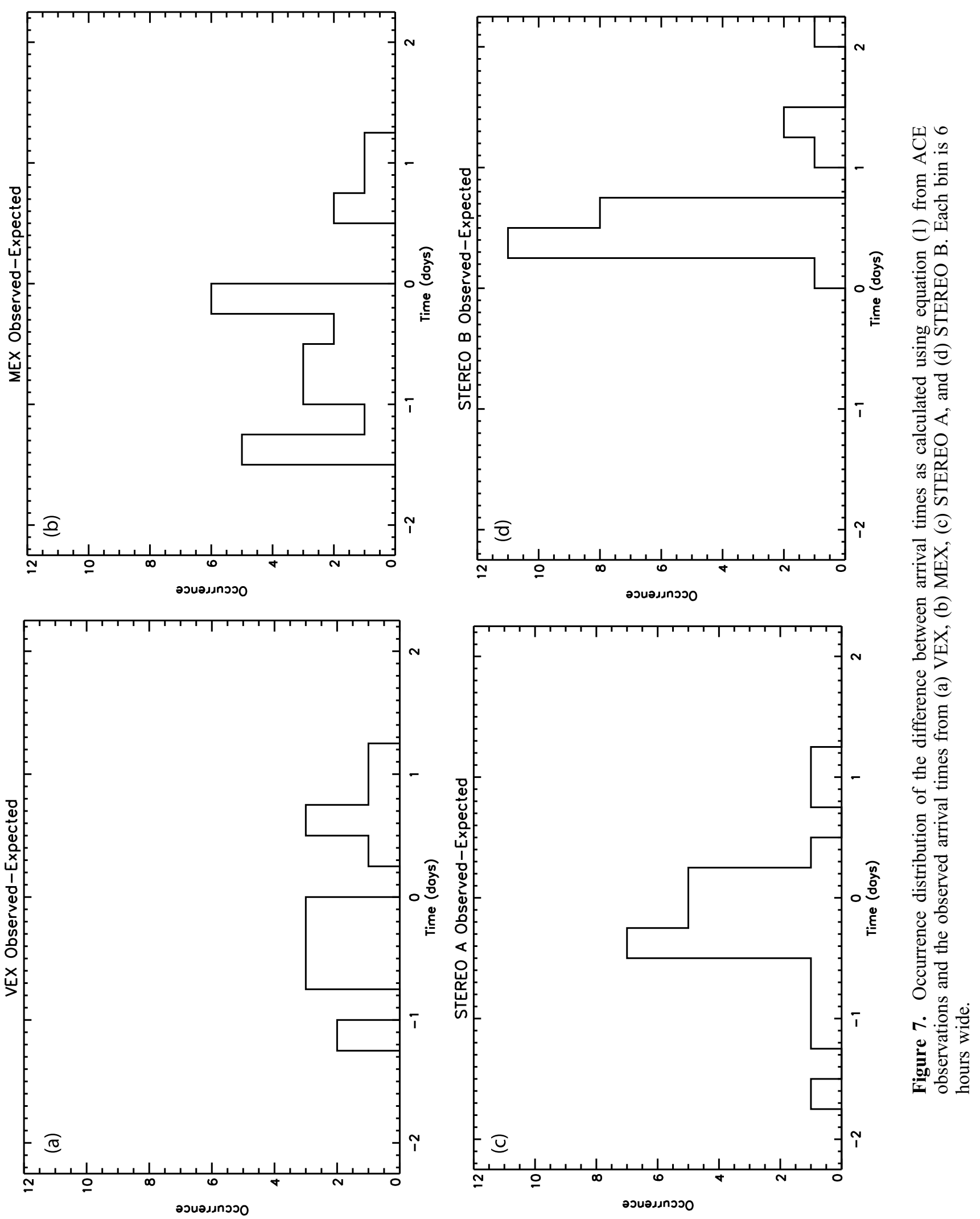
The difference is plotted in Figure 7 which shows a histogram with 6 hour bins. Figure 7 shows the difference between the expected and observed arrival times at VEX (Figure 7a), MEX (Figure 7b), STEREO A(Figure 7c) and STEREO B (Figure 7d). In Figure 7a we note that the majority of events occur within 1 day of the expected arrival time. However due to data gaps we could only get observations of 17 CIRs, although we note there could be other reasons for a lack of observations as noted below. From Figure $7 \mathrm{~b}$ we can see that 17 of the CIRs arrive within 1 day of when they are expected at MEX, with all CIRs arriving within 1.5 days of when they are expected. Looking at Figures $7 \mathrm{c}$ and $7 \mathrm{~d}$, where we have a more direct comparison of observations with ACE, we notice that the majority of CIRs arrive within 18 hours of when we expect. In the case of STEREO A, the distribution of time differences is mostly around zero with CIRs arriving both earlier and later than expected. However at STEREO B all CIRs arrive later than expected with the majority arriving 6-18 hours later than expected.

\section{Discussion}

[17] As shown by Figure 6 there is a discrepancy between arrival times of CIRs estimated from STEREO/HI J-maps and the observed arrival time at ACE. With regards to the estimates of arrival times at Venus and Mars both data sets have inherent problems. ACE is only one point in space and so cannot measure the radial CIR speed at all heliographic longitudes. Using only ACE gives us no information about the continuity of the CIR in longitude, although the addition of STEREO A and STEREO B in situ observations help to fill this gap. Observations of the source coronal hole on the Sun would also aid in establishing the continued presence of the CIR. Despite these potential drawbacks there is good agreement between the estimated arrival times and actual arrival times of CIRs at Mars using the limited data provided by ACE and two assumptions of CIR behavior.

[18] Estimates from STEREO/HI J-maps have their own inherent problems. While there have been several documented cases of the method used here providing velocities and directions in agreement with in situ data and estimates from other methods [Davis et al., 2010; Rouillard et al., 2009a, 2009b], other work has also shown that it can be difficult to identify complete events in the J-maps [Savani et al., 2009] and this can lead to an inaccurate estimation of the propagation angle or velocity or both. Indeed the correct identification of complete events for sufficient periods in the J-maps is probably the largest source of error for this method. In addition, the effects of the assumptions should be considered. This method assumes two things about the propagation of transient events through the STEREO/HI field of view, firstly that the transients are point-like objects and secondly that they move at a constant velocity and in a constant direction. The first assumption is an effect unique to the J-map method. CIRs are inherently three-dimensional objects, as is clear from their formation mechanism, and although they are visible in HI observations the tracking technique used here is not applicable as the tracking technique assumes point-like objects are being tracked. The extended nature of CIRs mean this technique is unsuitable for application to CIRs. However the signatures of plasma parcels which are entrained within a CIR can be treated as point-like objects and tracked using the method presented here, as highlighted in Figure 2 and described by Rouillard et al. [2009a]. By assuming a constant velocity and direction any acceleration which could alter the shape of the transient track is ignored. This can lead to an error in determining the velocity and direction which will lead to errors in determining the arrival time of the CIR front at other locations. If the direction of propagation is known from another method, the forward modeling method by Thernisien et al. [2009] for example, then a velocity profile can be found which might allow for an estimation with less error.

[19] There is one further source of error that should be considered when using the method of estimating solar transient event properties from STEREO/HI J-maps, which is the inherent user error in selecting the points for fitting to equation (2). This source of error has been more thoroughly investigated in previous work [Williams et al., 2009] using simulated point selection for an ideal trace and a Monte Carlo method to determine a suitable visibility of events in elongation to minimize errors.

[20] As mentioned in section 4 there are some events which were not observable in the VEX/ASPERA-4 data and, as previously described, the observations are intermittent due to the orbital configuration of VEX with respect to Venus. As VEX only spends a short time each orbit in the exosphere of Venus it is possible that ASPERA-4 was not in a good location for long enough to collect many clear signatures. Another reason for a lack of clear signatures could be due to the difference in densities in the atmospheres between Venus and Mars. It is possible that the increased density at Venus allows it to better retain the atmosphere during CIR transits. In addition, as hypothesized by Edberg et al. [2010], CIRs could be less well developed at the orbit of Venus and as such may have a smaller impact on the Venusian atmosphere.

[21] One potential further source of error that applies to all comparisons of data is the potential for latitudinal differences in the structure of interaction regions. Although the differences are small, approaching approximately $3^{\circ}$ at the end of July 2008 between ACE and either STEREO spacecraft, they could make a difference and could help to explain why the density structures observed at the 3 spacecraft are frequently so different. Helios observations [Schwenn and Marsch, 1990] showed that a latitudinal separation of only $1^{\circ}$ between two spacecraft can lead to significant observational differences as they observe different latitudinal slices of the same coronal hole. Indeed Simunac et al. [2009] undertook a correlation of expected bulk velocity and density at STEREO B from STEREO A during 2 events in July 2008 and while there was an approximately 1:1 correlation (0.96) in bulk velocity, there was a poor correlation in density.

\section{Conclusion}

[22] We have presented a technique for estimating the time of arrival of CIRs at planetary bodies and other spacecraft from observations of their passage through the inner heliosphere in the STEREO/HI instruments, and from ACE observations. As we have shown, using the mean measured velocity from ACE gives a good prediction. STEREO/HI will provide additional information about the 
temporal behavior and may in future give a predictive capability. At the time of writing the STEREO spacecraft are separated by approximately $70^{\circ}$ from Earth and so are moving out of position to get optimum views of Earth directed events. Hence predictions may need to be done by future missions. There are times when predictions of arrival times at Venus or Mars would only be possible in advance with STEREO/HI, such as when either planet is lagging the Earth in their orbit, as with Mars in Figure 1. In this case waiting for the CIR to arrive at ACE would be too late as it would have already arrived and impacted at Mars or Venus. STEREO/HI observations can still give us advance warning of an impending CIR at Earth if we use a reasonable estimate of the velocity, for example a mean velocity from several previous CIRs and make the assumption that the fitted angle is correct.

[23] Acknowledgments. A.O.W. is supported by STFC CASE studentship ST/F007612/1. N.J.T.E. was supported by the European 6th Framework, contract MEST-CT-2004-7512. S.E.M. and M.L. were supported by STFC rolling grant ST/H002480/1. The authors would also like to thank J. H. King and N. Papatashvilli at Adnet Systems, NASA GSFC, and CDAWeb for the data from the ACE spacecraft and for the STEREO merged 1 hourly in situ data. The authors would also like to acknowledge R. A. Harrison and C. J. Davis for the STEREO/HI images. Mars Express and Venus Express are ESA missions.

[24] Philippa Browning thanks Simon Plunkett and another reviewer for their assistance in evaluating this paper.

\section{References}

Barabash, S., et al. (2006), The analyzer of space plasmas and energetic atoms (ASPERA-3) for the Mars Express mission, Space Sci. Rev. 126(1-4), 113-164, doi:10.1007/s11214-006-9124-8.

Barabash, S., et al. (2007), The Analyzer of Space Plasmas and Energetic Atoms (ASPERA-4) for the Venus Express mission, Planet. Space Sci. 55(12), 1772-1792, doi:10.1016/j.pss.2007.01.014.

Cliver, E. (2006), The 1859 space weather event: Then and now, $A d v$. Space Res., 38(2), 119-129, doi:10.1016/j.asr.2005.07.077.

Cranmer, S. (2002), Coronal holes and the solar wind, in Multi-wavelength Observations of Coronal Structure and Dynamics, COSPAR Colloq. Ser., vol. 13, edited by P. C. H. Martens and D. P. Cauffman, pp. 3-12, Pergamon, Boston, Mass

Davies, J. A., R. A. Harrison, A. P. Rouillard, N. R. Sheeley Jr., C. H. Perry, D. Bewsher, C. J. Davis, C. J. Eyles, S. R. Crothers, and D. S. Brown (2009), A synoptic view of solar transient evolution in the inner heliosphere using the Heliospheric Imagers on STEREO, Geophys. Res. Lett., 36, L02102, doi:10.1029/2008GL036182.

Davis, C. J., J. Kennedy, and J. A. Davies (2010), Assessing the accuracy of CME speed and trajectory estimates from STEREO observations through a comparison of independent methods, Sol. Phys., 263(1-2), 209-222, doi:10.1007/s11207-010-9535-2.

Edberg, N. J. T., H. Nilsson, A. O. Williams, M. Lester, S. E. Milan, S. W. H. Cowley, M. Franz, S. Barabash, and Y. Futaana (2010), Pumping out the atmosphere of Mars through solar wind pressure pulses, Geophys. Res. Lett., 37, L03107, doi:10.1029/2009GL041814.

Eyles, C. J., et al. (2009), The Heliospheric Imagers onboard the STEREO mission, Sol. Phys., 254(2), 387-445, doi:10.1007/s11207-008-9299-0.

Galvin, A. B., et al. (2008), The Plasma and Suprathermal Ion Composition (PLASTIC) investigation on the STEREO observatories, Space Sci. Rev., 136(1-4), 437-486, doi:10.1007/s11214-007-9296-x.

Gosling, J., and V. Pizzo (1999), Formation and evolution of corotating interaction regions and their three dimensional structure, Space Sci. Rev., 89(1-2), 21-52, doi:10.1023/A:1005291711900.

Howard, R. A., et al. (2008), Sun Earth Connection Coronal and Heliospheric Investigation (SECCHI), Space. Sci. Rev., 136(1-4), 67-115, doi:10.1007/s11214-008-9341-4.

Lee, M. (2000), An analytical theory of the morphology, flows, and shock compressions at corotating interaction regions in the solar wind, J. Geophys. Res., 105(A5), 10,491-10,500.

Luhmann, J. G., A. Fedorov, S. Barabash, E. Carlsson, Y. Futaana, T. L. Zhang, C. T. Russell, J. G. Lyon, S. A. Ledvina, and D. A. Brain (2008a), Venus Express observations of atmospheric oxygen escape during the passage of several coronal mass ejections, J. Geophys. Res., 113, E00B04, doi:10.1029/2008JE003092.

Luhmann, J. G., et al. (2008b), STEREO IMPACT investigation goals, measurements, and data products overview, Space Sci. Rev., 136(1-4), 117-184, doi:10.1007/s11214-007-9170-x.

Mason, G. M., M. I. Desai, U. Mall, A. Korth, R. Bucik, T. T. von Rosenvinge, and K. D. Simunac (2009), In situ observations of CIRs on STEREO, Wind, and ACE during 2007-2008, Sol. Phys., 256(1-2), 393-408, doi:10.1007/ s11207-009-9367-0.

McComas, D., S. Bame, P. Barker, W. Feldman, J. Phillips, P. Riley, and J. Griffee (1998), Solar Wind Electron Proton Alpha Monitor (SWEPAM) for the Advanced Composition Explorer, Space Sci. Rev., 86(1-4), 563-612, doi:10.1023/A:1005040232597.

Milan, S. E., J. Hutchinson, P. D. Boakes, and B. Hubert (2009), Influences on the radius of the auroral oval, Ann. Geophys., 27(7), 2913-2924.

Richardson, I., and H. Cane (1995), Regions of abnormally low proton temperature in the solar wind (1965-1991) and their association with ejecta, J. Geophys. Res., 100(A12), 23,397-23,412.

Rouillard, A. P., et al. (2008), First imaging of corotating interaction regions using the STEREO spacecraft, Geophys. Res. Lett., 35, L10110, doi:10.1029/2008GL033767.

Rouillard, A. P., et al. (2009a), A multispacecraft analysis of a small-scale transient entrained by solar wind streams, Sol. Phys., 256(1-2), 307-326, doi:10.1007/s11207-009-9329-6.

Rouillard, A. P., et al. (2009b), A solar storm observed from the Sun to Venus using the STEREO, Venus Express, and MESSENGER spacecraft, J. Geophys. Res., 114, A07106, doi:10.1029/2008JA014034.

Rouillard, A. P., et al. (2010), Intermittent release of transients in the slow solar wind: 1. Remote sensing observations, J. Geophys. Res., 115, A04103, doi:10.1029/2009JA014471.

Savani, N. P., A. P. Rouillard, J. A. Davies, M. J. Owens, R. J. Forsyth, C. J. Davis, and R. A. Harrison (2009), The radial width of a coronal mass ejection between 0.1 and $0.4 \mathrm{AU}$ estimated from the Heliospheric Imager on STEREO, Ann. Geophys., 27(11), 4349-4358.

Schwenn, R., and E. Marsch (Eds.) (1990), Physics of the Inner Heliosphere, Phys. Chem. Space, vol. 20, Springer, New York.

Sheeley, N. R., Jr., J. Walters, Y. Wang, and R. Howard (1999), Continuous tracking of coronal outflows: Two kinds of coronal mass ejections, J. Geophys. Res., 104(A11), 24,739-24,767.

Sheeley, N. R., Jr., et al. (2008a), Heliospheric images of the solar wind at Earth, Astrophys. J., 675(1), 853-862.

Sheeley, N. R., Jr., et al. (2008b), SECCHI observations of the Sun's garden-hose density spiral, Astrophys. J., 674(2), L109-L112.

Simunac, K. D. C., L. M. Kistler, A. B. Galvin, M. A. Popecki, and C. J. Farrugia (2009), In situ observations from STEREO/PLASTIC: A test for L5 space weather monitors, Ann. Geophys., 27(10), 3805-3809.

Smith, C. W., J. L'Heureux, N. F. Ness, M. H. Acuña, L. F. Burlaga, and J. Scheifele (1998), The ACE magnetic fields experiment, Space Sci. Rev., 86(1-4), 613-632, doi:10.1023/A:1005092216668.

Tappin, S. J., and T. A. Howard (2009), Direct observation of a corotating interaction region by three spacecraft, Astrophys. J., 702(2), 862-870.

Thernisien, A., A. Vourlidas, and R. A. Howard (2009), Forward modeling of coronal mass ejections using STEREO/SECCHI data, Sol. Phys., 256(1-2), 111-130, doi:10.1007/s11207-009-9346-5.

Vennerstrom, S., N. Olsen, M. Purucker, M. Acuña, and J. Cain (2003), The magnetic field in the pile-up region at Mars, and its variation with the solar wind, Geophys. Res. Lett., 30(7), 1369, doi:10.1029/ 2003GL016883.

Wang, C., D. Du, and J. Richardson (2005), Characteristics of the interplanetary coronal mass ejections in the heliosphere between 0.3 and 5.4 AU, J. Geophys. Res., 110, A10107, doi:10.1029/2005JA011198.

Williams, A. O., J. A. Davies, S. E. Milan, A. P. Rouillard, C. J. Davis, C. H. Perry, and R. A. Harrison (2009), Deriving solar transient characteristics from single spacecraft STEREO/HI elongation variations: A theoretical assessment of the technique, Ann. Geophys., 27(12), 4359-4368.

Wood, B. E., R. A. Howard, A. Thernisien, and D. G. Socker (2010), The three-dimensional morphology of a corotating interaction region in the inner heliosphere, Astrophys. J., 708(2), L89-L94.

J. A. Davies, STFC Rutherford Appleton Laboratory, Didcot OX11 0QX, UK.

N. J. T. Edberg, Swedish Institute of Space Physics, PO Box 537, SE-75121 Uppsala, Sweden.

M. Fränz, Max Planck Institute for Solar System Research, D-37191 Katlenburg-Linden, Germany.

M. Lester, S. E. Milan, and A. O. Williams, Department of Physics and Astronomy, University of Leicester, Leicester LE1 7RH, UK. (aow2@ion. le.ac.uk) 